\title{
Stark shift and electric-field-induced dissociation of excitons in monolayer MoS2 and
} hBN/MoS2 heterostructures

\author{
Haastrup, Sten; Latini, Simone; Bolotin, Kirill; Thygesen, Kristian Sommer
}

Published in:

Physical Review B

Link to article, DOI:

10.1103/PhysRevB.94.041401

Publication date:

2016

Document Version

Publisher's PDF, also known as Version of record

Link back to DTU Orbit

Citation (APA):

Haastrup, S., Latini, S., Bolotin, K., \& Thygesen, K. S. (2016). Stark shift and electric-field-induced dissociation of excitons in monolayer MoS and hBN/MoS 2 heterostructures. Physical Review B, 94(4), [041401]. https://doi.org/10.1103/PhysRevB.94.0414012

\section{General rights}

Copyright and moral rights for the publications made accessible in the public portal are retained by the authors and/or other copyright owners and it is a condition of accessing publications that users recognise and abide by the legal requirements associated with these rights.

- Users may download and print one copy of any publication from the public portal for the purpose of private study or research.

- You may not further distribute the material or use it for any profit-making activity or commercial gain

- You may freely distribute the URL identifying the publication in the public portal 


\title{
Stark shift and electric-field-induced dissociation of excitons in monolayer $\mathrm{MoS}_{2}$ and $h \mathrm{BN} / \mathrm{MoS}_{2}$ heterostructures
}

\author{
Sten Haastrup, ${ }^{1}$ Simone Latini ${ }^{2,1}$ Kirill Bolotin, ${ }^{3,4}$ and Kristian S. Thygesen ${ }^{2,1}$ \\ ${ }^{1}$ Department of Physics, Center for Atomic-Scale Materials Design (CAMD), Technical University of Denmark, \\ 2800 Kgs. Lyngby, Denmark \\ ${ }^{2}$ Department of Physics, Center for Nanostructured Graphene (CNG), Technical University of Denmark, \\ 2800 Kgs. Lyngby, Denmark \\ ${ }^{3}$ Department of Physics, Vanderbilt University, Nashville Tennessee 37240, USA \\ ${ }^{4}$ Department of Physics, Freie University, 14195 Berlin, Germany
}

(Received 11 February 2016; published 1 July 2016)

\begin{abstract}
Efficient conversion of photons into electrical current in two-dimensional semiconductors requires, as a first step, the dissociation of the strongly bound excitons into free electrons and holes. Here we calculate the dissociation rates and energy shift of excitons in monolayer $\mathrm{MoS}_{2}$ as a function of an applied in-plane electric field. The dissociation rates are obtained as the inverse lifetime of the resonant states of a two-dimensional hydrogenic Hamiltonian which describes the exciton within the Mott-Wannier model. The resonances are computed using complex scaling, and the effective masses and screened electron-hole interaction defining the hydrogenic Hamiltonian are computed from first principles. For field strengths above $0.1 \mathrm{~V} / \mathrm{nm}$ the dissociation lifetime is shorter than $1 \mathrm{ps}$, which is below the lifetime associated with competing decay mechanisms. Interestingly, encapsulation of the $\mathrm{MoS}_{2}$ layer in just two layers of hexagonal boron nitride $(h \mathrm{BN})$, enhances the dissociation rate by around one order of magnitude due to the increased screening. This shows that dielectric engineering is an effective way to control exciton lifetimes in two-dimensional materials.
\end{abstract}

DOI: 10.1103/PhysRevB.94.041401

Two-dimensional (2D) semiconductors, such as singleand few-layer transition-metal dichalcogenides, are presently being intensively researched due to their extraordinary electronic and optical properties which include strong lightmatter interactions, spin-valley coupling, and easily tunable electronic states [1-14]. One of the hallmarks of the 2D semiconductors is the presence of strongly bound excitons with binding energies reaching up to $30 \%$ of the band gap. These large binding energies are mainly a result of the reduced dielectric screening in two dimensions [15-19]. Although such strongly bound excitons are highly interesting from a fundamental point of view (for example, in the context of Bose-Einstein condensates [20]) they are problematic for many of the envisioned applications of 2D materials, such as photodetectors and solar cells which rely on efficient conversion of photons into electrical currents. This is because the strong attraction between the electron and the hole makes it difficult to dissociate the excitons into free carriers.

Photocurrent measurements on suspended $\mathrm{MoS}_{2}$ samples have found that the photocurrent produced by below-band-gap photons is strongly dependent on the applied voltage indicating that the electric field plays an important role in the generation of free carriers [21]. One way to increase the photoresponse could be to embed the active 2D material into a van der Waals heterostructure [22-24]. This embedding would enhance the screening of the electron-hole interaction without altering the overall shape of the band structure of the material. The effects of this increased screening on the exciton dissociation are studied in this Rapid Communication.

In general, rigorous calculations of exciton binding energies require a many-body approach, such as the Bethe-Salpeter equation (BSE) which directly finds the (real) poles of the interacting response function, corresponding to the neutral excitation energies of the system $[25,26]$. Such calculations are computationally demanding and typically only used to study excitations from the ground state, i.e., not in the presence of external fields. We mention, however, that the BSE has been used to study field-induced exciton dissociation in carbon nanotubes by fitting the BSE absorption spectrum to the Fano line shape [27]. In this Rapid Communication we take a different approach using that, under certain simplifying circumstances, the calculation of the many-body excitonic state can be reformulated as an effective hydrogenic Hamiltonian whose eigenvalues and eigenstates represent the exciton binding energies and the envelope wave function describing the relative electron-hole motion. This is the so-called Mott-Wannier model which has been instrumental in the description of excitons in inorganic bulk semiconductors. A $2 \mathrm{D}$ version of the Mott-Wannier model has recently been shown to yield exciton binding energies in good agreement with BSE calculations and experiments for both freestanding $[15,16,18,19,28]$ and supported $[15,28,29]$ transition-metal dichalcogenide layers. The dissociation rate of the exciton is then obtained by complex scaling, which is a formally exact technique to compute resonance energies and lifetimes. By employing a recently developed quantum-classical method for calculating the dielectric function of general van der Waals heterostructures, we predict the effect of embedding the $\mathrm{MoS}_{2}$ in $h \mathrm{BN}$ on the screened electron-hole interaction and exciton dissociation rate.

When an in-plane constant electric field is applied to an exciton, it will eventually decay into a free electron and hole. This effect belongs to a class first studied by Keldysh [30] and Franz [31], who examined how the optical properties of semiconductors change in the presence of a static electric field. The application of a constant electric field changes the exciton from a bound state to a resonance with a finite lifetime equal to the inverse dissociation rate. 
The literature on resonances in quantum physics is vast, and we will not go into the topic here but simply mention a few important facts. First, it should be understood that even the definition of a resonance is nontrivial. The reason for this can be understood from Howland's razor which states that no satisfactory definition of a resonance can depend only on the structure of a single operator on an abstract Hilbert space [32]. To illustrate the content of the statement consider the Stark effect in hydrogen: Let $\hat{H}(\epsilon)=-\frac{1}{2} \Delta-1 / r+\epsilon x$. It can be shown that $\hat{H}(\epsilon)$ is unitarily equivalent to $\hat{H}\left(\epsilon^{\prime}\right)$ for all nonzero $\epsilon$ and $\epsilon^{\prime}$ s. Since we expect the properties of the resonances and, in particular, their lifetimes to depend on field strength $\epsilon$, this example shows that the resonance cannot be viewed only as a property of the operator $\hat{H}(\epsilon)$. Instead the notion of resonance is only meaningful when the real-space geometry of the given system and relevant boundary conditions on the wave functions are considered.

There are generally two approaches used to compute resonances. The so-called indirect methods identify resonances as the poles of the scattering amplitude analytically extended to the complex energy plane [33], whereas the direct methods obtain the resonance states directly as eigenstates of a complex scaled non-Hermitian Hamiltonian [34,35]. In this Rapid Communication we will use the latter approach.

To describe excitons in a 2D semiconductor we use a MottWannier model of the form

$$
\left[-\frac{\nabla_{2 \mathrm{D}}^{2}}{2 \mu_{\mathrm{ex}}}+W(\mathbf{r})\right] F(\mathbf{r})=E_{\mathrm{b}} F(\mathbf{r}),
$$

where $\mu_{\mathrm{ex}}$ is the exciton effective mass $\mu_{\mathrm{ex}}^{-1}=m_{\mathrm{e}}^{-1}+m_{\mathrm{h}}^{-1}$, $W$ is the screened electron-hole interaction, $\mathbf{r}$ is an in-plane position vector, and $E_{\mathrm{b}}$ denotes the exciton binding energy. In principle there should be an exchange term included here, but a full $a b$ initio solution of the BSE has shown that the exchange term decreases the binding energy of the lowest exciton in
$\mathrm{MoS}_{2}$ by less than 4\% [15], and the term can therefore be neglected.

The screened electron-hole interaction is obtained as the inverse Fourier transform of $\left[\epsilon_{2 \mathrm{D}}(\mathbf{q}) q\right]^{-1}$, where $\epsilon_{2 \mathrm{D}}(\mathbf{q})$ is the static dielectric function of the $2 \mathrm{D}$ material and $1 / q$ is the in-plane 2D Fourier transform of $1 / r$. In the small- $q$ limit, we can approximate $\epsilon$ as a linear function of $q$ [16-19] so that

$$
\epsilon_{2 \mathrm{D}}(\mathbf{q})=1+2 \pi \alpha q,
$$

with $\alpha$ being the polarizability of the material. An analytic expression can then be obtained for the screened electron-hole interaction [17],

$$
W(\mathbf{r})=\frac{1}{4 \alpha}\left[Y_{0}(x)-H_{0}(x)\right]_{x=r / 2 \pi \alpha},
$$

where $Y_{0}$ is a Bessel function of the second kind and $H_{0}$ is a Struve function. For later use we note that both of these functions are analytic on the entire complex plane away from $z=0$.

The expression (3) for the screened interaction relies on a first-order expansion of $\epsilon_{2 \mathrm{D}}(q)$ around $q=0$; the validity of this approximation has been demonstrated for a number of freestanding 2D semiconductors [16,18,19] and recently for $\mathrm{MoS}_{2}$ embedded in a few layers of $h \mathrm{BN}$ [15]. As a rule of thumb, the linear screening approximation [Eq. (2)] remains valid for intralayer excitons in van der Waals heterostructures as long as the in-plane exciton radius is large compared to the thickness of the heterostructure [15]. For thicker slabs, the linear approximation breaks down, and the fully $q$-dependent $\epsilon_{2 \mathrm{D}}(\mathbf{q})$ must be used to obtain $W(r)$. We follow the common practice of using the static dielectric function for evaluating the screened interaction of the Mott-Wannier model. For details on how we calculate the dielectric functions of 2D layers and heterostructures we refer to Ref. [29]. Using these methods, the static dielectric function $\epsilon_{2 \mathrm{D}}(q)$ can be calculated, and the slope at $q=0$ can be determined. (a) 893938988888888

(b)

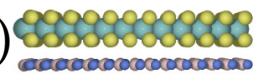

(c) 809900808989

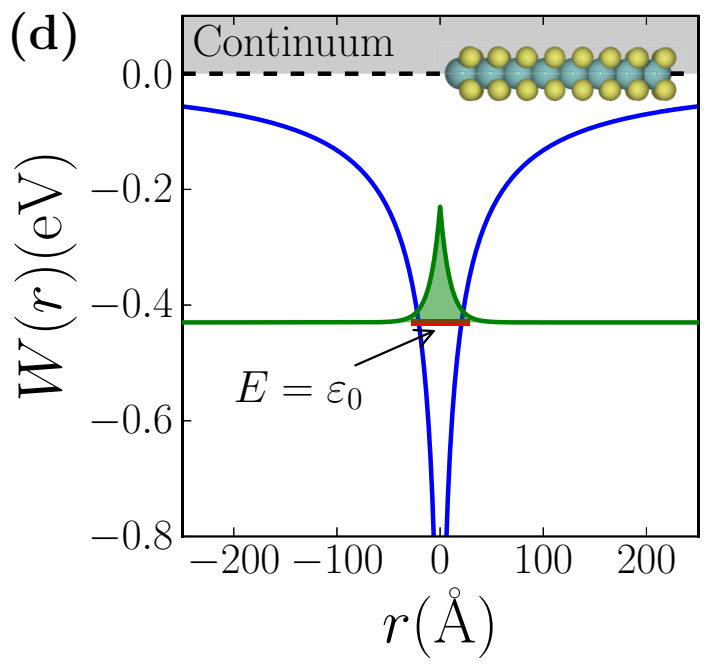

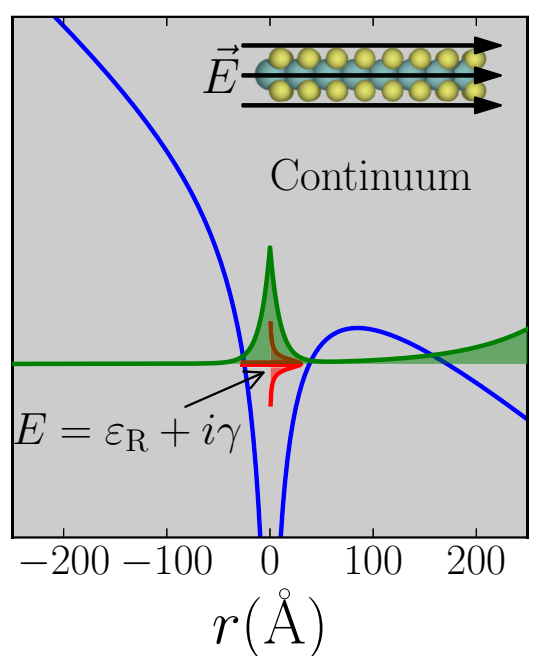

FIG. 1. (a)-(c) The three different structures considered in this Rapid Communication: isolated $\mathrm{MoS}_{2}, \mathrm{MoS}_{2}$ on a single layer of $h \mathrm{BN}$, and $\mathrm{MoS}_{2}$ sandwiched between two $h \mathrm{BN}$ layers. (d) Illustration of the Mott-Wannier model for monolayer MoS ${ }_{2}$ in the absence (left) and presence (right) of an in-plane constant electric field. The exciton potential is shown in blue, the exciton wave function is sketched in green, and the energy is shown in red. When an electric field is applied, the energy of the exciton shifts down, and the sharp energy peak is broadened due to the coupling to the continuum of states. 
Here we have considered a $\mathrm{MoS}_{2}$ layer in three different configurations: isolated, placed on a single layer of $h \mathrm{BN}$, and sandwiched between two $h \mathrm{BN}$ layers. The systems are sketched in Figs. 1(a)-1(c). The distance between the $\mathrm{MoS}_{2}$ base plane and the $h \mathrm{BN}$ sheets was $5.1 \AA$ and was chosen as the mean of the interlayer distance in pure $\mathrm{MoS}_{2}$ and $h \mathrm{BN}$. Sensitivity testing showed that varying this distance by $20 \%$ results in a variation in the slope of $\epsilon_{2 \mathrm{D}}(\mathbf{q})$ of less than $2 \%$.

The lattices of $\mathrm{MoS}_{2}$ and $h \mathrm{BN}$ are incommensurable, but the quantum-electrostatic heterostructure model introduced in Ref. [29] allows us to obtain the dielectric function of the heterostructure by electrostatic coupling of the response of the individual layers thus avoiding the issue of in-plane lattice mismatch. Table I shows the obtained polarizabilities and corresponding exciton binding energies. As expected, embedding the $\mathrm{MoS}_{2}$ in $h \mathrm{BN}$ leads to an increase in screening and a reduction in the binding energy with the calculated results for the binding energy being in good agreement with $a b$ initio calculations [15].

Once an in-plane constant electric field is applied to the system, the bound states of the Mott-Wannier Hamiltonian become metastable. The situation is illustrated in Fig. 1(d). In the model we have used, we assume that the band structure and, in particular, the effective mass of the exciton are not altered by the electric field.

Within the so-called direct methods, a resonance is defined as an eigenstate of the Hamiltonian under the boundary condition that only outgoing waves exist outside the scattering region. Such an eigenstate must necessarily have a complex eigenvalue $E=\epsilon_{0}-i \gamma$ and a wave function that adopts the asymptotic form $e^{ \pm i K x}$ for $x \rightarrow \pm \infty$ (focusing on the one-dimensional case for simplicity) where $K=k-i \kappa$ with $k>0$ (an outgoing wave) and $\kappa>0$. The latter condition implies that the wave function increases exponentially away from the scattering region. The decay rate of the resonance state, evaluated as the rate of decay of the probability for finding the particle in any finite region of space, is given by $\gamma=k \kappa$. It can be shown that the resonance eigenvalue $E$ is a pole of the analytically continued scattering matrix [36].

To compute the resonance, one could in principle solve the Schrödinger equation with the appropriate boundary conditions. In practice, however, it is more convenient to perform a "complex scaling" of the Hamiltonian, whereby the coordinate $r \rightarrow e^{i \theta} r$ and $\nabla \rightarrow e^{-i \theta} \nabla$, and then solve for the eigenstates of the resulting (non-Hermitian) operator $\hat{H}_{\theta}$ with the more standard zero boundary conditions. For $\theta>\tan ^{-1}(\gamma / k)$, the complex scaled resonance wave function (that is the wave function evaluated on the line $r e^{i \theta}$ after analytic continuation) is an eigenstate of $\hat{H}_{\theta}$ with eigenvalue $E$ but now decaying

TABLE I. Calculated values for the polarizability $(\alpha)$ and exciton binding energy $\left(E_{\mathrm{b}}\right)$ for single-layer $\mathrm{MoS}_{2}$ in the three configurations shown in Figs. 2(a)-2(c).

\begin{tabular}{lcc}
\hline \hline Material & $\alpha$ (a.u.) & $E_{\mathrm{b}}(\mathrm{eV})$ \\
\hline $\mathrm{MoS}_{2}$ & 11.1 & 0.62 \\
$\mathrm{MoS}_{2}-h \mathrm{BN}$ & 13.0 & 0.55 \\
$h \mathrm{BN}-\mathrm{MoS}_{2}-h \mathrm{BN}$ & 16.1 & 0.47 \\
\hline \hline
\end{tabular}

exponentially as $r \rightarrow \pm \infty$. The resonances thus appear as isolated complex eigenvalues of $\hat{H}_{\theta}$ with energy independent of $\theta$ and a square integrable wave function [37]. The complex scaled wave function of the bound states remain exponentially decaying eigenstates of $\hat{H}_{\theta}$ with real eigenvalues [34].

The unbound continuum states have a different behavior: If the potentials involved are localized, the asymptotic form of these states as $r \rightarrow \infty$ is $e^{i k r}$ with $k, r \in \mathbb{R}$. They are thus finite at infinity but non-normalizable. If this is to remain true after the complex scaling is performed, the transformation $r \rightarrow r e^{i \theta}$ must be accompanied by the transformation $k \rightarrow k e^{-i \theta}$. As the energy of a plane wave is proportional to $k^{2}$, the complex scaling operation results in the energy of the continuum states rotating into the complex plane at an angle of $2 \theta$.

We mention that the complex scaling procedure cannot be applied to any potential $V(\mathbf{r})$ [35], but the class of potentials for which the procedure works is large enough to include the bare and the screened Coulomb potential [38] as well as a constant electric field [39].

In Fig. 2 we show an example of the spectrum of the complex-scaled exciton Hamiltonian for isolated $\mathrm{MoS}_{2}$ in zero field for different values of the scaling parameter $\theta$. The two classes of states, bound and unbound, can clearly be distinguished; for zero field there are no resonances.

For the systems shown in Figs. 2(a)-2(c) we compute the screened interaction between charges located in the $\mathrm{MoS}_{2}$ layer using the random phase approximation (RPA) and the local density approximation (LDA) as implemented in the GPAW code $[40,41]$. The response calculations were done with a $60 \times$ $60 k$-point grid and a 150-eV energy cutoff for $\mathbf{G}$ and $\mathbf{G}^{\prime}$. The bandstructure obtained from the LDA calculations gives an effective exciton mass for $\mathrm{MoS}_{2}$ of $0.27 m_{\mathrm{e}}$. Once $\alpha$ and $\mu_{\mathrm{ex}}$ are known, the $2 \mathrm{D}$ eigenvalue problem for the complexscaled Hamiltonian is solved on a real-space grid using radial coordinates. In order to converge the exciton energies, a large simulation cell is needed-significantly larger than the exciton radius, which is around $10 \AA$ for all of the systems considered.

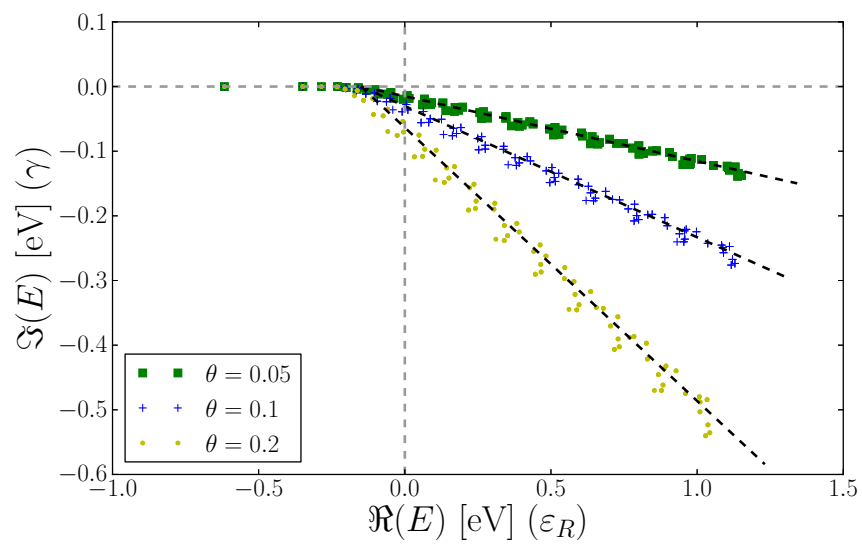

FIG. 2. The different behaviors of bound and continuum states under the complex scaling operation for the potential corresponding to isolated $\mathrm{MoS}_{2}$. The black dashed lines start at $-0.15 \mathrm{eV}$ and have been rotated into the complex plane by $-2 \theta$ for each of the complex scaling angles. Note that the continuum starts at $-0.15 \mathrm{eV}$ and not 0 because of the finite size of the simulation box. 


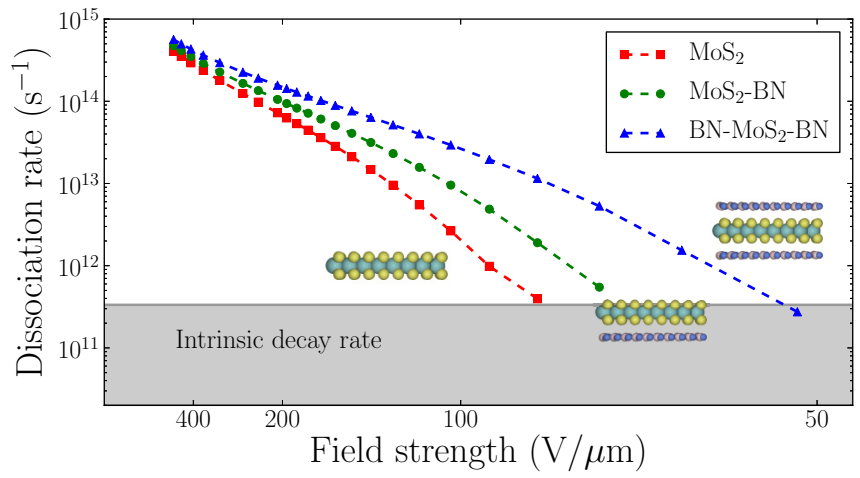

FIG. 3. The dissociation rate of an exciton in the $\mathrm{MoS}_{2}$ layer as a function of in-plane field strength for the three different structures. The intrinsic decay rate spans between the defect-assisted fast decay of the excitons of $2-5$ ps (upper limit) and the much slower radiative recombination of the excitons at room temperature (lower limit).

As the screened potential has a logarithmic singularity at $r=0$ while being virtually flat at the edge of the simulation cell, a nonlinear grid is used, which allows us to perform simulations in a disk of radius $250 \AA$. The Laplacian is represented by a finite-difference stencil. In order to avoid diagonalization of the full Hamiltonian, we used the iterative eigensolver ARPACK.

Figure 3 shows the $\mathrm{MoS}_{2}$ exciton dissociation rate as a function of in-plane field strength for three different structures. As expected, larger fields lead to shorter lifetimes, and the rate is seen to depend roughly exponentially on $1 / E$ for the considered field strengths. It can also be seen that the dissociation rate can be tuned to a high degree by changing the environment of the $\mathrm{MoS}_{2}$. When $\mathrm{MoS}_{2}$ is placed on a single layer of boron nitride, the extra screening greatly increases the dissociation rate, and similarly, when the $\mathrm{MoS}_{2}$ is sandwiched between two layers of $\mathrm{BN}$, the rate is even larger. This is as expected since larger screening results in more weakly bound excitons, which should in turn dissociate more readily. Adding more $h \mathrm{BN}$ layers on either side is expected to further enhance the screening and hence the dissociation rates, but this has not been pursued here as the linear screening model breaks down in this regime [15].

Along with information about the lifetime of the resonant states, the complex eigenvalue can provide information on the Stark shift of the resonance energy, an effect which is directly observable in optical absorption measurements. Figure 4 shows how the real part of the eigenvalue varies with field strength, and as expected, for small fields we observe a parabolic shift. The breakdown of this parabolic behavior occurs at smallest fields for the most screened excitons.

Recently, it has been shown that excitons in 2D materials can be described by a 2D hydrogen model with an effective dielectric constant [28], which for the linear screening described by Eq. (2) is given by $\epsilon_{\mathrm{eff}}=\frac{1}{2}+\frac{1}{2} \sqrt{1+32 \pi \alpha \mu / 3}$. Based on this model and second-order perturbation theory, the shift can be predicted to be

$$
\Delta E=-\frac{21}{64} \frac{\epsilon_{\mathrm{eff}}^{4}}{\mu^{3}} E^{2} .
$$

Figure 4 shows that this prediction fits well with our calculations for small fields.

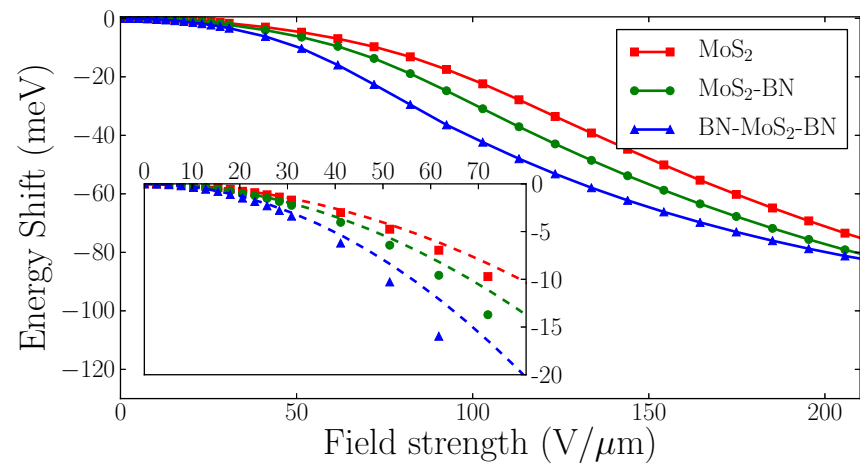

FIG. 4. The Stark shift in the $\mathrm{MoS}_{2}$ heterostructures. The inset shows the shift for small fields, along with the shift predicted for a 2D hydrogen atom with an effective dielectric constant $\epsilon_{\text {eff }}$; see Eq. (4).

In a real device, the field-induced dissociation of excitons described here is in competition with other decay mechanisms, such as direct radiative recombination [42], defect-assisted recombination [43], and exciton-exciton annihilation [44]. The relative importance of these effects is highly dependent on the temperature of the $\mathrm{MoS}_{2}$, the presence and concentration of defects, and the exciton density.

At very low temperatures, the direct radiative decay of zero momentum excitons dominates with a characteristic lifetime of $\sim 200 \mathrm{fs}[42,45,46]$. At room temperature, most of the excitons have nonvanishing momenta, and the radiative recombination lifetime is $\sim 1 \mathrm{~ns}[42,43]$. For these systems, defect-assisted recombination therefore becomes an important mechanism with a characteristic lifetime of $2-5$ ps $[43,47,48]$. Exciton-exciton annihilations become important only when the density of excitons in a sample is large; equivalently when the average distance between excitons is small. At a density of $1 \times 10^{12} \mathrm{~cm}^{-2}$, the effective lifetime from annihilation is on the order of $10 \mathrm{ps}$ [44].

The calculations performed here indicate that for field strengths larger than $0.1 \mathrm{~V} / \mathrm{nm}$, the dissociation lifetime is shorter than 1 ps in all the systems considered. A potential gradient of this size $(0.1 \mathrm{~V} / \mathrm{nm})$ over the extent of the exciton (around $2 \mathrm{~nm}$ ) is realistic to achieve close to the metal-MoS $\mathrm{M}_{2}$ contact region where charge transfer and interface dipole formation driven by Fermi-level mismatch can lead to significant variation of the potential and band energies even in the absence of an applied bias voltage. Under such conditions, the field-induced dissociation is faster than any other decay channel and should therefore dominate as indicated by the fact that in Fig. 3, the data points all lie above the shaded region.

To summarize we have used complex scaling to compute the lifetime of excitons in two-dimensional $\mathrm{MoS}_{2}$ and $\mathrm{MoS}_{2} / \mathrm{hBN}$ structures under an applied static electric field. The exciton was simulated using a 2D Mott-Wannier model which has previously been found to yield a reliable description of the lowestlying excitonic states in transition-metal dichalcogenides. We found that for field strengths around $0.1 \mathrm{~V} / \mathrm{nm}$, the exciton dissociation is larger than the intrinsic exciton decay rate in $\mathrm{MoS}_{2}$. Moreover, encapsulation in a few layers of $h \mathrm{BN}$ was found to increase the dissociation rate by an order of magnitude for fixed field strength due to the increased screening provided by the electrons in the $h \mathrm{BN}$. 
The authors gratefully acknowledge financial support from the Center for Nanostructured Graphene (Project No.
DNRF103) financed by the Danish National Research Foundation.
[1] Q. H. Wang, K. Kalantar-Zadeh, A. Kis, J. N. Coleman, and M. S. Strano, Nat. Nanotechnol. 7, 699 (2012).

[2] K. F. Mak, C. Lee, J. Hone, J. Shan, and T. F. Heinz, Phys. Rev. Lett. 105, 136805 (2010)

[3] A. Splendiani, L. Sun, Y. Zhang, T. Li, J. Kim, C.-Y. Chim, G. Galli, and F. Wang, Nano Lett. 10, 1271 (2010).

[4] A. Ramasubramaniam, Phys. Rev. B 86, 115409 (2012).

[5] D. Y. Qiu, F. H. da Jornada, and S. G. Louie, Phys. Rev. Lett. 111, 216805 (2013).

[6] M. M. Ugeda, A. J. Bradley, S.-F. Shi, H. Felipe, Y. Zhang, D. Y. Qiu, W. Ruan, S.-K. Mo, Z. Hussain, Z.-X. Shen et al., Nat. Mater. 131091 (2014).

[7] K. He, N. Kumar, L. Zhao, Z. Wang, K. F. Mak, H. Zhao, and J. Shan, Phys. Rev. Lett. 113, 026803 (2014).

[8] D. Jariwala, V. K. Sangwan, L. J. Lauhon, T. J. Marks, and M. C. Hersam, ACS Nano 8, 1102 (2014).

[9] M. Bernardi, M. Palummo, and J. C. Grossman, Nano Lett. 13, 3664 (2013).

[10] O. Lopez-Sanchez, D. Lembke, M. Kayci, A. Radenovic, and A. Kis, Nat. Nanotechnol. 8, 497 (2013).

[11] J. S. Ross, P. Klement, A. M. Jones, N. J. Ghimire, J. Yan, D. Mandrus, T. Taniguchi, K. Watanabe, K. Kitamura, W. Yao et al., Nat. Nanotechnol. 9, 268 (2014).

[12] A. Pospischil, M. M. Furchi, and T. Mueller, Nat. Nanotechnol, 9, 257 (2014).

[13] K. F. Mak, K. He, C. Lee, G. H. Lee, J. Hone, T. F. Heinz, and J. Shan, Nature Mater. 12, 207 (2013).

[14] A. Chernikov, T. C. Berkelbach, H. M. Hill, A. Rigosi, Y. Li, O. B. Aslan, D. R. Reichman, M. S. Hybertsen, and T. F. Heinz, Phys. Rev. Lett. 113, 076802 (2014).

[15] S. Latini, T. Olsen, and K. S. Thygesen, Phys. Rev. B 92, 245123 (2015).

[16] T. C. Berkelbach, M. S. Hybertsen, and D. R. Reichman, Phys. Rev. B 88, 045318 (2013).

[17] P. Cudazzo, C. Attaccalite, I. V. Tokatly, and A. Rubio, Phys. Rev. Lett. 104, 226804 (2010).

[18] P. Cudazzo, I. V. Tokatly, and A. Rubio, Phys. Rev. B 84, 085406 (2011).

[19] O. Pulci, P. Gori, M. Marsili, V. Garbuio, R. Del Sole, and F. Bechstedt, Europhys. Lett. 98, 37004 (2012).

[20] M. M. Fogler, L. V. Butov, and K. S. Novoselov, Nat. Commun. 5, 4555 (2014).

[21] A. R. Klots, A. K. M. Newaz, B. Wang, D. Prasai, H. Krzyzanowska, J. Lin, D. Caudel, N. J. Ghimire, J. Yan, B. L. Ivanov, K. A. Velizhanin, A. Burger, D. G. Mandrus, N. H. Tolk, S. T. Pantelides, and K. I. Bolotin, Sci. Rep. 46608 (2014).
[22] H. Terrones, F. López-Urías, and M. Terrones, Sci. Rep. 31549 (2013).

[23] L. Britnell, R. Ribeiro, A. Eckmann, R. Jalil, B. Belle, A Mishchenko, Y.-J. Kim, R. Gorbachev, T. Georgiou, S. Morozov et al., Science 340, 1311 (2013).

[24] A. Geim and I. Grigorieva, Nature (London) 499, 419 (2013).

[25] G. Strinati, Phys. Rev. B 29, 5718 (1984).

[26] G. Onida, L. Reining, and A. Rubio, Rev. Mod. Phys. 74, 601 (2002).

[27] V. Perebeinos and P. Avouris, Nano Lett. 7, 609 (2007).

[28] T. Olsen, S. Latini, F. Rasmussen, and K. S. Thygesen, Phys. Rev. Lett. 116, 056401 (2016).

[29] K. Andersen, S. Latini, and K. S. Thygesen, Nano Lett. 15, 4616 (2015).

[30] L. V. Keldysh, J. Exp. Theor. Phys. 33, 994 (1957).

[31] W. Franz, Z. Naturforschung A 13a, 484 (1958).

[32] B. Simon, Int. J. Quantum Chem. 14, 529 (1978).

[33] J. Taylor, Scattering Theory : The Quantum Theory on Nonrelativistic Collisions (Wiley, New York, 1972), p. 477.

[34] E. Balslev and J. M. Combes, Commun. Math. Phys. 22, 280 (1971).

[35] J. Aguilar and J. M. Combes, Commun. Math. Phys. 22, 269 (1971).

[36] N. Hatano, K. Sasada, H. Nakamura, and T. Petrosky, Prog. Theor. Phys. 119, 187 (2008).

[37] W. P. Reinhardt, Annu. Rev. Phys. Chem. 33, 223 (1982).

[38] B. Simon, Ann. Math. 97, 247 (1973).

[39] I. W. Herbst and B. Simon, Phys. Rev. Lett. 41, 1759 (1978).

[40] J. J. Mortensen, L. B. Hansen, and K. W. Jacobsen, Phys. Rev. B 71, 035109 (2005).

[41] J. Enkovaara, C. Rostgaard, J. J. Mortensen, J. Chen, M. Dulak, L. Ferrighi, J. Gavnholt, C. Glinsvad, V. Haikola, H. A. Hansen et al., J. Phys.: Condens. Matter 22, 253202 (2010).

[42] M. Palummo, M. Bernardi, and J. C. Grossman, Nano Lett. 15, 2794 (2015).

[43] H. Shi, R. Yan, S. Bertolazzi, J. Brivio, B. Gao, A. Kis, D. Jena, H. G. Xing, and L. Huang, ACS Nano 7, 1072 (2013).

[44] D. Sun, Y. Rao, G. A. Reider, G. Chen, Y. You, L. Brézin, A. R. Harutyunyan, and T. F. Heinz, Nano Lett. 14, 5625 (2014).

[45] H. Wang, C. Zhang, W. Chan, C. Manolatou, S. Tiwari, and F. Rana, Phys. Rev. B 93, 045407 (2016).

[46] C. Poellmann, P. Steinleitner, U. Leierseder, P. Nagler, G. Plechinger, M. Porer, R. Bratschitsch, C. Schueller, T. Korn, and R. Huber, Nature Mater. 14, 889 (2015).

[47] T. Korn, S. Heydrich, M. Hirmer, J. Schmutzler, and C. Schüller, Appl. Phys. Lett. 99, 102109 (2011).

[48] D. Lagarde, L. Bouet, X. Marie, C. R. Zhu, B. L. Liu, T. Amand, P. H. Tan, and B. Urbaszek, Phys. Rev. Lett. 112, 047401 (2014). 